\title{
Higher-Order Permanent Scatterers Analysis
}

\author{
Alessandro Ferretti \\ Tele-Rilevamento Europa S.r.l. (TRE), via Vittoria Colonna 7, 20149 Milano, Italy \\ Dipartimento di Elettronica e Informazione, Politecnico di Milano, Piazza Leonardo da Vinci 32, 20133 Milano, Italy \\ Email: alessandro.ferretti@treuropa.com
}

\author{
Marco Bianchi \\ Tele-Rilevamento Europa S.r.l. (TRE), via Vittoria Colonna 7, 20149 Milano, Italy \\ Email:marco.bianchi@treuropa.com
}

\section{Claudio Prati}

Dipartimento di Elettronica e Informazione, Politecnico di Milano, Piazza Leonardo da Vinci 32, 20133 Milano, Italy Email:prati@elet.polimi.it

\author{
Fabio Rocca \\ Dipartimento di Elettronica e Informazione, Politecnico di Milano, Piazza Leonardo da Vinci 32, 20133 Milano, Italy \\ Email: rocca@elet.polimi.it
}

Received 25 September 2004; Revised 27 December 2004

\begin{abstract}
The permanent scatterers (PS) technique is a multi-interferogram algorithm for DInSAR analyses developed in the late nineties to overcome the difficulties related to the conventional approach, namely, phase decorrelation and atmospheric effects. The successful application of this technology to many geophysical studies is now pushing toward further improvements and optimizations. A possible strategy to increase the number of radar targets that can be exploited for surface deformation monitoring is the adoption of parametric super-resolution algorithms that can cope with multiple scattering centres within the same resolution cell. In fact, since a PS is usually modelled as a single pointwise scatterer dominating the background clutter, radar targets having cross-range dimension exceeding a few meters can be lost (at least in C-band datasets), due to geometrical decorrelation phenomena induced in the high normal baseline interferograms of the dataset. In this paper, the mathematical framework related to higher-order SAR interferometry is presented as well as preliminary results obtained on simulated and real data. It is shown how the PS density can be increased at the price of a higher computational load.
\end{abstract}

Keywords and phrases: RADAR remote sensing, synthetic aperture RADAR, interferometric applications, permanent scatterer analysis.

\section{INTRODUCTION}

Differential SAR interferometry (DInSAR) is a remote sensing technology capable of measuring possible displacements of radar targets along the line of sight (LOS) by computing the difference of the phase values of two SAR scenes gathered at different times over the same area of interest $[1,2,3,4,5]$. As well known, interferometric data can be used to recover high-resolution topographic profiles (acquiring data pairs from slightly different looking angles) [6] or to highlight possible surface deformation phenomena (compensating the

This is an open access article distributed under the Creative Commons Attribution License, which permits unrestricted use, distribution, and reproduction in any medium, provided the original work is properly cited. phase data for the local topography and the two acquisition geometries) [1]. Since SAR systems operate in the microwave domain (typically the operating frequency is within the $1-10 \mathrm{GHz}$ band), even subcentimetre range variations generate phase shifts that can be detected by the sensor, thus providing —at least theoretically — a powerful tool for precise geodetic surveys over large areas.

During the last decade the availability of an increasing number of satellite SAR data suitable for interferometric applications, and in particular the historical archive built by the ESA missions ERS-1 (1991-2001), ERS-2 (1995), and Envisat (2001), created major interest in different scientific communities $[7,8,9,10]$. First successes in detecting and measuring surface deformation phenomena from space, related to volcanic activity [7] or seismic-related events [8], 
were welcome by geophysicists, volcanologists, and seismologists as a big step toward the development of new earlywarning tools based on remote-sensed data. However, later studies highlighted also the limits of DInSAR technology, and dampened somewhat the initial enthusiasm of both the geophysical and remote-sensing communities $[2,5]$. Problems related to temporal and geometrical decorrelation (i.e., reflectivity changes as a function of time and incidence angle of the acquisition [11]) as well as atmospheric artefacts (due to the different tropospheric and ionospheric conditions at the time of the SAR acquisitions [5]) were encountered in almost all real-life applications, pushing toward the development of more sophisticated techniques aimed at getting precise and reliable displacement information at least on a subset of image pixels $[12,13,14,15,16,17,18]$.

Whenever enough images are available, DInSAR limitations can be overcome by adopting a multi-interferogram framework. The permanent scatterers (PS) technique $[12,13$, 14], developed in the late nineties at Politecnico di Milano, takes advantage of long temporal series of SAR data, acquired over the area of interest along the same (nominal) satellite orbit, to filter out atmospheric artefacts and to identify a subset of image pixels where high-precision measurements can be carried out. These pixels, almost unaffected by temporal and geometrical decorrelation (usually but not necessarily corresponding to man-made objects), are called permanent scatterers (PS) $[12,13]$. The technique has been applied successfully to a number of applications from subsidence [13] and volcano monitoring [19] to slow-landslide detection $[12,20]$ and is currently used for both research and commercial activities.

The PS analysis can be divided into two processing steps. First the so-called atmospheric phase screen (APS) is estimated and removed from every interferogram; this task can be performed by exploiting the different statistical behaviours of the atmospheric and motion phase components $[12,13,14]$. Then a pixel-by-pixel analysis is carried out, searching for all PS available in the area of interest and jointly estimating their elevation (with respect to the local ellipsoid) and the time series of their LOS displacements.

Although the algorithms adopted for the first processing phase (i.e., APS removal) are rather complex, the mathematical framework used for the estimation of the unknown parameters (i.e., PS elevation and motion components) is simple. All interferograms are generated, after data resampling, using a common master scene selected within the dataset available. Each interferogram is characterized by a temporal and geometrical baseline. The PS is modelled as a dominant pointwise scatterer within its resolution cell, unaffected by temporal and geometrical decorrelation. Interferometric phase values can then be easily related to PS elevation (via the normal baseline of the interferogram) and target displacement (via the temporal baseline) [12].

Due to the adoption of a first-order scattering-centre model, amplitude data relative to the same image pixel are not used in the analysis, since they are supposed to be independent of time and aspect angle of the acquisition. This simplifies the mathematical framework, since it involves (wrapped) phase data only and reduces the computational load. Of course different models, usually polynomial, can be applied to the displacement time series (phase data), depending on the application at hand and the number of available images. A PS is said to be detected whenever the dispersion of the phase residues with respect to the phase model is below a certain threshold.

A possible limitation of this strategy is related to the model adopted for the detection of coherent targets (i.e., targets unaffected by phase decorrelation). Since baseline dispersion of satellite datasets available today is usually greater than $400 \mathrm{~m} \mathrm{[14],} \mathrm{coherent} \mathrm{radar} \mathrm{targets} \mathrm{having} \mathrm{cross-range}$ dimension greater than a few meters, for C-band sensors [12], can be lost (i.e., not labelled as PS), even though their radar signature does not actually change with time, that is, no temporal decorrelation phenomena affect the target. Reflectivity changes as a function of the variations of the looking angle of the SAR acquisition (proportional to the normal baseline) can then limit the number of measurement points identified by the conventional PS analysis, especially in unfavorable datasets characterised by high dispersion of the normal baseline values.

In this paper, a possible generalization of the framework adopted by the PS approach, and more generally to multiinterferogram DInSAR analysis, is presented, allowing the extraction of more information relative to the area of interest. Instead of considering a single scattering centre, higher-order models involving two or more scattering centres within the resolution cell are adopted (super-resolution framework), thus relaxing the geometrical constraints imposed to the radar target to behave as a PS. For the sake of simplicity, this paper will focus on the second-order model (two scattering centres), although the same mathematical framework, with minor changes, can be extended to higher-order models.

This approach is somewhat complimentary to the tomographic formulation presented in [21] using airborne SAR data. The multibaseline tomographic SAR processing discussed in [21] is an algorithm to recover a three-dimensional (3D) image from a set of 2D acquisitions gathered over the same area from slightly different looking angles, based on the projection slice theorem and a Fourier analysis. The following sections present a procedure aiming at the same goal, but adopting a parametric model where no data interpolation is carried out before the estimation of the unknown parameters. It should be noted that the scattering-centre representation is often used by the radar community, in particular for radar target identification [22], and it is therefore a rather natural option in processing microwave images. Moreover, the superiority of parametric methods for solving layover effects from complex topography has already been highlighted in the numerical simulations carried out in $[23,24]$.

This paper is organized as follows. In Section 2, the mathematical framework and the novel model for PS analysis, along with the notation adopted through the paper, are presented. Section 3 is devoted to the solution of the inverse problem related to the estimation of the unknown parameters of the scattering centres, outlining a possible strategy to cope with overfitting problems and model order selection. 
Sections 4 and 5 describe the results obtained using simulated and real data respectively. Finally, Section 6 gives the conclusions and summarizes ongoing efforts.

\section{SIGNAL MODEL}

Let $N+1$ be the number of SAR images available acquired over the same area of interest with a common acquisition geometry. Following the PS approach presented in $[12,13,14]$, data are first coregistered on a unique master and $N$ differential interferograms between all SAR images and the master are computed using a reference digital elevation model (DEM) of the area (at worst just the WGS84 ellipsoid) and the satellite state vectors describing the acquisition geometry of the multitemporal dataset. For the sake of simplicity, we will suppose that atmospheric phase components superimposed on the data have been successfully removed using one of the strategies proposed in $[12,13]$, however similar considerations hold for phase differences between two nearby pixels, where the impact of APS is strongly reduced due to its low-frequency behaviour (the correlation length of the APS is usually greater than $1-2 \mathrm{~km} \mathrm{[5]).}$

\section{1. $\quad$ First-order model}

According to the hypothesis that a PS can be modelled as a single dominant target within the SAR resolution cell, the amplitude value of the signal backscattered by the target is independent of time and looking angle of the SAR acquisition, while the phase value $\phi$ of the $i$ th interferogram relative to pixel $P$ in the image can be modeled as

$$
\phi\left(P, t_{i}\right)=\mu\left(P, t_{i}\right)+C_{\mathrm{DEM}}^{i} \mathcal{E}(P)+\eta\left(P, t_{i}\right), \quad i=1, \ldots, N,
$$

where $t_{i}$ is the temporal baseline of the $i$ th interferogram, $\mu\left(P, t_{i}\right)$ is the phase component due to a possible LOS displacement of the PS, $\eta\left(P, t_{i}\right)$ takes into account phase noise and any atmospheric leakage, $\varepsilon(P)$ is the PS elevation with respect to the reference DEM, and $C_{\mathrm{DEM}}^{i}$ is proportional to the normal baseline $B_{i}$ of the interferogram:

$$
C_{\mathrm{DEM}}^{i}=\frac{4 \pi B_{i}}{\lambda R \sin \theta} \equiv \frac{A_{\mathrm{DEM}}^{i}}{\sin \theta},
$$

where $\lambda$ is the wavelength of the sensor $(5.6 \mathrm{~cm}$ for ESA and RADARSAT sensors), $R$ is the sensor-to-target distance, and $\theta$ is the local incidence angle of the illuminating radiation. The $A_{\mathrm{DEM}}^{i}$ coefficient in the equation above will be used later on.

Time series analysis of the first term in (1) should reflect target motion. To simplify the notation, but without loss of generality, in the following a constant-velocity model will be adopted (a uniform strain rate hypothesis is often used in geophysical modelling):

$$
\mu\left(P, t_{i}\right)=\frac{4 \pi}{\lambda} v(P) t_{i}=C_{v}^{i} v(P)
$$

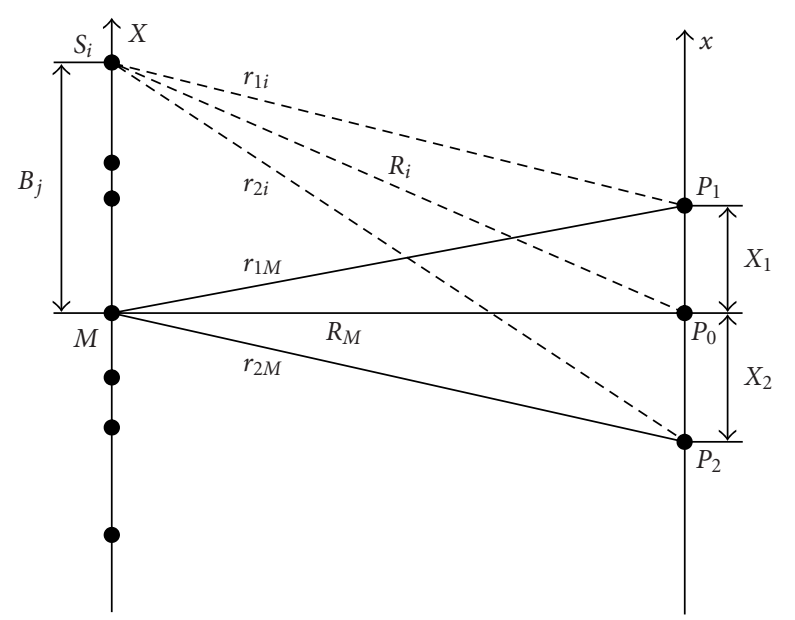

FIGURE 1: Geometrical schematization of the problem under study. An $N$-sensor array receives signal from two (or more) sources. $B$ and $x$ are cross-range coplanar axes normal to the azimuth direction (see also Figure 2).

where $v(P)$ is the velocity of the scattering centre within the resolution cell.

From the above considerations, we can finally write the model of the interferometric phase of each interferogram:

$$
\phi\left(P, t_{i}\right)=C_{v}^{i} v(P)+C_{\mathrm{DEM}}^{i} \varepsilon(P)+\eta\left(P, t_{i}\right), \quad i=1, \ldots, N .
$$

As discussed in $[12,13]$, the two unknown parameters $v$ and $\varepsilon$ can be estimated jointly maximizing the (first-order) phase coherence function:

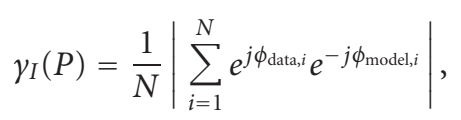

where $\phi_{\text {model, } i}$ is computed according to (4). Coherence values range from 0 to 1 as a function of the dispersion of the phase residues with respect to the model $(\eta)$. From (5) it is clear that no use of the amplitude data is present in the estimation of $v$ and $\varepsilon$ in standard PS analysis.

\subsection{Second-order model}

We will now discuss the case of two dominant scatterers within the same resolution cell. This yields a second-order model, first outlined in [25]. The mathematical framework can be made very similar to that adopted in direction of arrival (DOA) analyses (see $[23,24,26]$ and references therein). The multibaseline dataset relative to pixel $P$ can be viewed as a snapshot of a nonuniform array composed of $N$ sensors (Figures 1 and 2).

Here $X, x$, and $R_{M}$ are coplanar axes belonging to a plane normal to the satellite trajectories (related to the azimuth direction) and passing through the resolution cell under study. $M$ is the position of the master acquisition, $S_{i}$ is the projection of the $i$ th slave pass on the $X$-axis. $B_{i}$ is then the corresponding normal baseline. Point $P_{0}$ represents the reference 


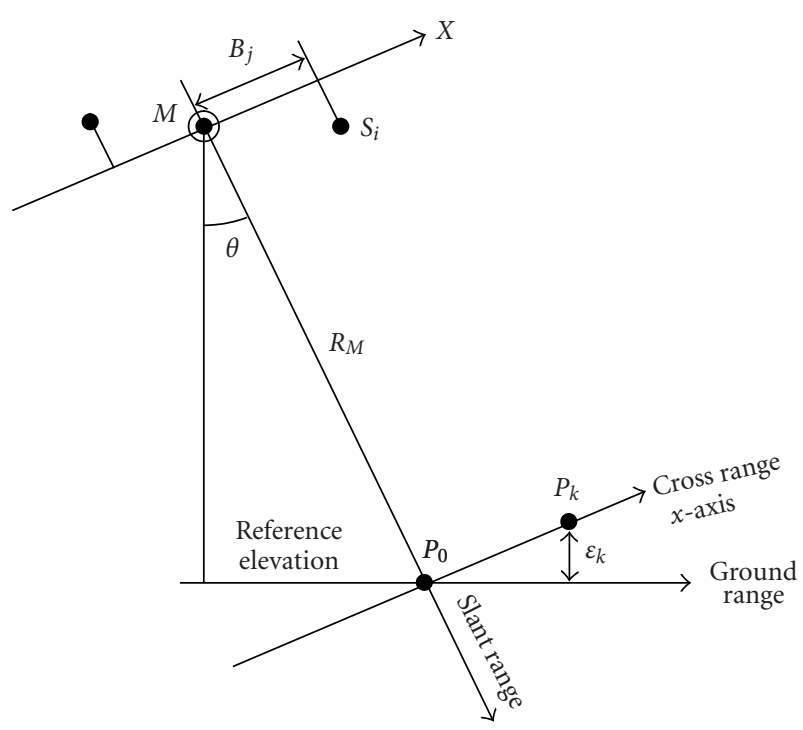

FIgURE 2: SAR acquisition geometry. $M$ is the master antenna, $S$ the slave. Parallel baseline components do not impact the mathematical modelling for phase variations. $P_{0}$ represents the reference DEM for the pixel under analysis. $P$ is the dominant scattering-centre within the resolution cell.

topography (i.e., reference DEM). The complex signal received by the $i$ th sensor can be modeled as

$$
s_{i}=z_{1} e^{j(4 \pi / \lambda) r\left(B_{i}, t_{i}, x_{1}\right)}+z_{2} e^{j(4 \pi / \lambda) r\left(B_{i}, t_{i}, x_{2}\right)},
$$

where the dependency of the range coordinate on the normal baseline $B_{i}$, the temporal baseline $t_{i}$, and the cross-range position $x_{k}$ of scatterer $k$ has been highlighted, and $z_{1}$ and $z_{2}$ are the complex reflectivities of the two scattering centres.

For the sake of simplicity, in this paper we will suppose that all scatterers within the same resolution cell are affected by the same displacement. In particular, considering the constant velocity model, we will suppose

$$
v_{1}=v_{2}=v
$$

From Figure 1 it is easy to verify that

$$
\begin{aligned}
r_{k i} & =r\left(B_{i}, t_{i}, x_{k}\right)=\sqrt{R_{M}^{2}+\left(B_{i}-x_{k}\right)^{2}} \\
& \cong R_{M}+\frac{\left(B_{i}-x_{k}\right)^{2}}{2 R_{M}}, \quad k=1,2
\end{aligned}
$$

where the approximation is valid for satellite sensors (typically $R_{M}>800 \mathrm{~km}$ and $|B|<2 \mathrm{~km}$ ). In particular, for the master acquisition, we have

$$
r_{k M} \cong R_{M}+\frac{x_{k}^{2}}{2 R_{M}}, \quad k=1,2 .
$$

If now we define the differential interferogram as

$$
y_{i} \equiv \frac{s_{M} s_{i}^{*}}{\left|s_{M}\right|} e^{-j(4 \pi / \lambda)\left(R_{M}-R_{i}\right)}
$$

(where $s^{*}$ is the complex conjugate of $s$ ), the absolute value of the interferogram equals the amplitude of the slave image and it is easy to demonstrate (see the appendix) that (2), (6), (8), (9), and (10), jointly with hypothesis (7), lead to the expression

$$
y_{i}=\beta_{1} e^{j\left(A_{\mathrm{DEM}}^{i} x_{1}-C_{v}^{i} v\right)}+\beta_{2} e^{j\left(A_{\mathrm{DEM}}^{i} x_{2}-C_{v}^{i} v\right)}, \quad i=1, \ldots, N
$$

where

$$
\begin{aligned}
& \beta_{1}=\frac{\left|z_{1}\right|^{2}+z_{2} z_{1}^{*} e^{j(4 \pi / \lambda)\left(x_{2}^{2}-x_{1}^{2} / R_{M}\right)}}{\left|s_{M}\right|} \\
& \beta_{2}=\frac{\left|z_{2}\right|^{2}+z_{1} z_{2}^{*} e^{j(4 \pi / \lambda)\left(x_{1}^{2}-x_{2}^{2} / R_{M}\right)}}{\left|s_{M}\right|} .
\end{aligned}
$$

It is worth noting that

$$
\left|\beta_{1}\right|^{2}=\left|z_{1}\right|^{2}, \quad\left|\beta_{2}\right|^{2}=\left|z_{2}\right|^{2} \text {. }
$$

Moreover, since $x=\varepsilon / \sin \theta$ (Figure 2), system (11) can also be written as

$$
y_{i}=\beta_{1} e^{j\left(C_{\mathrm{DEM}}^{i} \varepsilon_{1}-C_{v}^{i} v\right)}+\beta_{2} e^{j\left(C_{\mathrm{DEM}}^{i} \varepsilon_{2}-C_{v}^{i} v\right)} .
$$

Equations (11) and (12) show that, by introducing a secondorder model for the scattering and a constant velocity model for target displacement, data can be modelled as the sum of two complex sinusoids characterized by 5 unknown parameters ( 3 real and 2 complex variables) to be estimated by the dataset available: $v, x_{1}, x_{2}, \beta_{1}, \beta_{2}$.

\subsection{Higher-order models}

The generalization to higher-order models is straightforward. In general, for a scattering model of order $K$, where $x_{k}$ is the position of the $k$ th target on the $x$-axis in Figure 1 $(k=1, \ldots, K)$, we can generalize expression (11) as follows:

$$
y_{i}=e^{-j C_{v}^{i} v}\left[\sum_{m=1}^{K} \beta_{m} e^{j A_{\mathrm{DEM}}^{i} x_{k}}\right],
$$

where

$$
\beta_{m}=\frac{1}{\left|s_{M}\right|} \sum_{l=1}^{K} z_{l} z_{m}^{*} e^{j(4 \pi / \lambda)\left(x_{l}^{2}-x_{m}^{2} / 2 R_{M}\right)}
$$

Again, the data can be modelled as a sum of $K$ complex sinusoids of unknown amplitudes and frequencies. As already mentioned, in the following sections we will focus on the second-order model, but extension to higher-order analyses does not impact the mathematical framework. 


\section{INVERSE PROBLEM SOLUTION}

As discussed in the previous section, the adoption of a second-order model for PS analysis introduces three further unknown parameters with respect to the standard approach, even considering scatterers affected by the same displacement. In order to estimate $v, x_{1}, x_{2}, \beta_{1}, \beta_{2}$, the nonlinear model (11) needs to be inverted on a pixel-by-pixel basis. Although most of the procedures used in DOA problems require uniform array $[23,24,26,27,28]$, many algorithms can be applied to solve this problem. Here we adopt the nonlinear least squares method (NLSM) presented in [27], since it turned out to be robust and effective for the problem under study. However, future research efforts will address the selection of the best inversion algorithm for real data, taking into account accuracy, precision, robustness performance, as well as the related computational load. Let $\boldsymbol{\theta}$ be a $p \times 1$ vector of unknown parameters, $x(\boldsymbol{\theta})$ the model $(N$-dimensional nonlinear function of the parameters vector), and $\mathbf{y}$ the data vector. As is well known, the object function used in NLSM is

$$
J=[\mathbf{y}-x(\boldsymbol{\theta})]^{H}[\mathbf{y}-x(\boldsymbol{\theta})] .
$$

For the problem under study we have

$$
x(m)=\sum_{k=1}^{n} \alpha_{k} e^{j\left(\omega_{k} m+\varphi_{k}\right)} .
$$

In order to recover the unknown pulse frequencies $\left\{\omega_{k}\right\} \in$ $[-\pi, \pi]$, amplitudes $\left\{\alpha_{k}\right\}$, and initial phases $\left\{\varphi_{k}\right\}$ starting from the observed data $y$, the following cost function has to be minimized:

$$
J=\sum_{i=1}^{N}\left|y_{i}-\sum_{k=1}^{n} \alpha_{k} e^{j\left(\omega_{k} i+\varphi_{k}\right)}\right|^{2} .
$$

We now introduce the following notation [27]:

$$
\begin{aligned}
& \beta_{k}=\alpha_{k} e^{j \varphi_{k}}, \\
& \boldsymbol{\beta}=\left[\beta_{1} \cdots \beta_{n}\right]^{T}, \\
& \mathbf{B}=\left[\begin{array}{ccc}
e^{j \omega_{1}} & \cdots & e^{j \omega_{n}} \\
\vdots & & \vdots \\
e^{j N \omega_{1}} & \cdots & e^{j N \omega_{n}}
\end{array}\right] .
\end{aligned}
$$

The model is then linear in $\boldsymbol{\beta}$ and nonlinear in $\boldsymbol{\omega}$ :

$$
\mathbf{x}=\mathbf{B} \beta,
$$

and the cost function is now

$$
J=(\mathbf{Y}-\mathbf{B} \boldsymbol{\beta})^{H}(\mathbf{Y}-\mathbf{B} \boldsymbol{\beta}),
$$

where $\mathbf{Y}=[y(1) \cdots y(N)]^{T}$. B is a Vandermonde matrix that fulfils the following rank property:

$$
\operatorname{rank}(\mathbf{B})=n \quad \text { if } N \geq n, \quad \omega_{k} \neq \omega_{p} \quad \text { for } k \neq p .
$$

If conditions (24) are met, the matrix $\left(\mathbf{B}^{H} \mathbf{B}\right)^{-1}$ exists and (23) yields

$$
\begin{aligned}
J= & {\left[\boldsymbol{\beta}-\left(\mathbf{B}^{H} \mathbf{B}\right)^{-1} \mathbf{B}^{H} \mathbf{Y}\right]^{H}\left[\mathbf{B}^{H} \mathbf{B}\right]\left[\boldsymbol{\beta}-\left(\mathbf{B}^{H} \mathbf{B}\right)^{-1} \mathbf{B}^{H} \mathbf{Y}\right] } \\
& +\mathbf{Y}^{H} \mathbf{Y}-\mathbf{Y}^{H} \mathbf{B}\left(\mathbf{B}^{H} \mathbf{B}\right)^{-1} \mathbf{B}^{H} \mathbf{Y} .
\end{aligned}
$$

For each choice of $\boldsymbol{\omega}=\left[\omega_{1}, \ldots, \omega_{n}\right]^{T}$ in $\mathbf{B}$ (with $\omega_{k} \neq$ $\omega_{p}$ for $k \neq p$ ) a vector $\beta$ that cancels the first term of (25) can be found. Vectors $\boldsymbol{\beta}$ and $\boldsymbol{\omega}$ minimizing expression (25) are therefore,

$$
\begin{gathered}
\widehat{\boldsymbol{\omega}}=\underset{\omega}{\operatorname{argmax}}\left[\mathbf{Y}^{H} \mathbf{B}\left(\mathbf{B}^{H} \mathbf{B}\right)^{-1} \mathbf{B}^{H} \mathbf{Y}\right], \\
\hat{\boldsymbol{\beta}}=\left.\left(\mathbf{B}^{H} \mathbf{B}\right)^{-1} \mathbf{B}^{H} \mathbf{Y}\right|_{\boldsymbol{\omega}=\hat{\boldsymbol{\omega}}} .
\end{gathered}
$$

The application of the NLSM to system (11) leads to the following cost function:

$$
J=\sum_{i=1}^{N}\left|y_{i}-\sum_{k=1}^{2} \beta_{k} e^{j \omega_{k_{i}}}\right|^{2},
$$

where $\beta_{k}$ is defined by (12), $\omega_{k_{i}}=C_{\mathrm{DEM}}^{i} \varepsilon_{k}+C_{v}^{i} v$, and $N$ is the number of data available. $\mathbf{B}$ is then an $(N \times 2)$ matrix:

$$
\mathbf{B}=\left[\begin{array}{cc}
e^{j\left(C_{\mathrm{DEM}}^{1} \varepsilon_{1}-C_{v}^{1} v\right)} & e^{j\left(C_{\mathrm{DEM}}^{1} \varepsilon_{2}-C_{v}^{i} v\right)} \\
\vdots & \vdots \\
e^{j\left(C_{\mathrm{DEM}}^{N} \varepsilon_{1}-C_{v}^{N} v\right)} & e^{j\left(C_{\mathrm{DEM}}^{N} \varepsilon_{2}-C_{v}^{N} v\right)}
\end{array}\right] .
$$

For each matrix B expression (26) must be evaluated and the values $\left(\hat{\varepsilon}_{1}, \hat{\varepsilon}_{2}, \hat{v}\right)$ maximizing it are the solution. Figure 3 shows an example of target function to be maximized in (26): local maxima are present (the problem is strongly nonlinear), but whenever the signal-to-noise ratio (SNR) is high enough and the underlying model fits the data, the global maximum is well pronounced.

Once we have estimated deformation rate and elevations of the scatterers, we can estimate $\hat{\beta}_{1}$ and $\hat{\beta}_{2}$ via (27). The estimated values $\hat{v}, \hat{\varepsilon}_{1}, \hat{\varepsilon}_{2}, \hat{\beta}_{1}, \hat{\beta}_{2}$ can then be used to build

$$
\hat{y}_{i}=\hat{\beta}_{1} e^{j\left(C_{\mathrm{DEM}}^{i} \hat{\varepsilon}_{1}-C_{v}^{i} \hat{v}\right)}+\hat{\beta}_{2} e^{j\left(C_{\mathrm{DEM}}^{i} \hat{\varepsilon}_{2}-C_{v}^{i} \hat{v}\right)}, \quad i=1, \ldots, N,
$$

and to define the "second-order phase coherence," natural extension of the first-order coherence (5):

$$
\gamma_{I I}(P)=\frac{1}{N}\left|\sum_{i=1}^{N} e^{j \phi_{\text {data, },}} e^{-j<\hat{y}_{i}}\right|,
$$

where $\angle \hat{y}_{i}$ is the phase value of expression (30). Similarly to $\gamma_{I}$, the accent in (31) is posed on the dispersion of the phase 


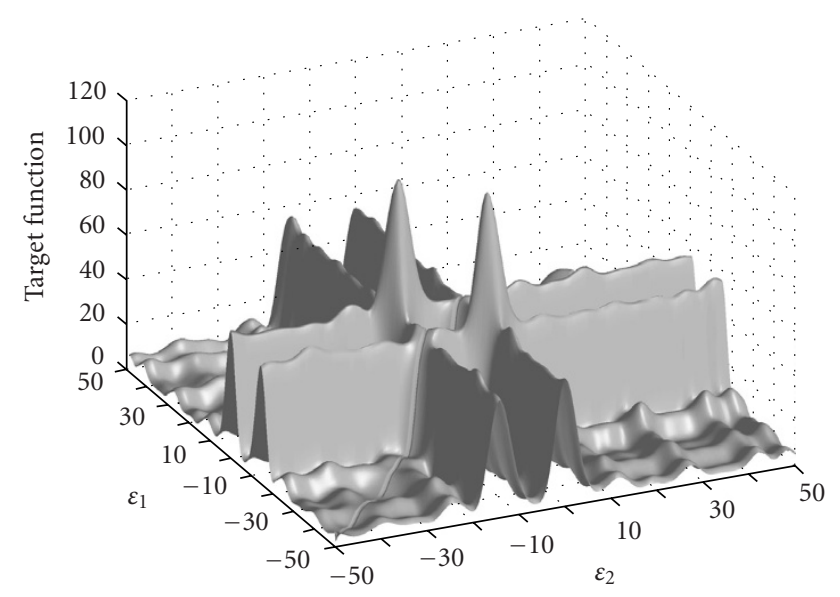

FIgURE 3: Example of target function to be maximized in (26). For visualization purposes, the velocity parameter is fixed to the optimum value while $\varepsilon_{1}$ and $\varepsilon_{2}$ vary in the range $[-50,50]$ meters. This is a real data example, referring to the dataset analysed in Section 5. The two maxima (the function is symmetric) are easily detectable.

residues rather than the LMS error between the complex data vector and the model. This is not unreasonable whenever the final target of the analysis is the extraction of precise displacement time series (related to phase data) rather than the characterization of local reflectivity. Of course, since the computational load of this optimization is proportional to the volume of the three-dimensional parameter space in which the unknowns $v, \varepsilon_{1}, \varepsilon_{2}$ can vary (plausibility region), the number of operations per pixel is much higher than in conventional PS analysis, where a portion of a two-dimensional space is spanned by the estimation algorithm $[12,13]$.

The solution $\hat{\varepsilon}_{1}=\hat{\varepsilon}_{2}$ causes the product $B^{H} B$ to be a singular matrix and expression (26) will diverge. Thus, the case of a single scatterer will not be solved by the second-order model as two coinciding scattering centres but the first-order scatterer will be split into a dominant scatterer and a second very low-reflectivity centre. This yields an overblown solution which causes

$$
\gamma_{I I}(P)<\gamma_{I}(P) .
$$

The pixel will consequently be considered a first-order scatterer.

\subsection{Model order selection}

Common to all parametric analyses, model order selection (MOS) is a key step that should be carefully studied before accepting the results of the estimation. Indeed, although the condition $\gamma_{I I}>\gamma_{I}$ is generally satisfied, this does not necessarily imply that the second order is a better model for the data.

As well known, one of the most difficult and critical issues facing sensor arrays systems is the detection of the number of sources impinging on the array [26]. Problems of model order selection are usually faced based on the Akaike information criteria [29] or the Rissanen minimum description length criteria [30].

It should be noted that a simple MOS strategy such as the computation of the periodogram for each pixel, looking for the number of maxima, would not be successful, due to resolution limits. In fact, two nearby scattering centres (just a few meters apart) are not detectable by nonparametric approaches.

By limiting the analysis on first- and second-order models only (the estimation is to be carried out on millions of pixels in a typical SAR scene and so the computational burden is a key issue for applications), the problem can be cast as a hypothesis testing procedure. In fact, we can formulate two mutually exclusive options for the scattering mechanism:

\section{$H_{0}$ : resolution cell $P$ contains one scattering-centre, \\ $H_{1}$ : resolution cell $P$ contains two (or more) \\ scattering-centres.}

In other words, after first- and second-order analysis, we have to establish whether or not $H_{1}$ can be accepted, fixing a confidence level for the decision. In general, in order to limit overfitting, $H_{1}$ hypothesis should be accepted (or analogously $H_{0}$ should be rejected) only if the probability that the data vector comes from a single scattering centre is below a certain threshold THR, that is, if

$$
\operatorname{Pr}\left(H_{0} \mid \text { data }\right)<\mathrm{THR} \Longrightarrow H_{1} .
$$

The key issue is then the definition of the rejection region. Intuitively, one should accept $H_{1}$ only "if it is worth," for example, if the dispersion of the phase residues is significantly reduced adopting a more complex model, or-equivalentlyif the gain in phase coherence is high enough. Although an analytical analysis is very complex, a possible solution can be achieved using a Monte Carlo approach, discussed in the next section.

\section{NUMERICAL SIMULATION}

First- and second-order estimation algorithms were tested first on simulated numerical data at different levels of signalto-noise ratio (SNR). The simulation allows one to better appreciate the limits related to the first-order model. Two data vectors are reported in Figures 3 and 4. In both cases two motionless scattering centres are considered $(v=0$ and $H_{1}=$ true). The baseline distribution used in the simulation resembles that of the real dataset available, described in Section 5. No noise is present in these examples, so the data satisfy (11), (12), and (13).

First we notice that whenever two identical scatterers (i.e., $z_{1}=z_{2}$ ) are present (Figure 4 ), the first-order model discussed in Section 2.1 can fit the data almost perfectly. In fact, apart from a modulation of the amplitude values as a function of the normal baseline, phase values are identical to that originated by a single point scatterer positioned in the "electromagnetic barycentre" of the structure under study. This can be generalized to the $K$-scatterer model. A mirror, 

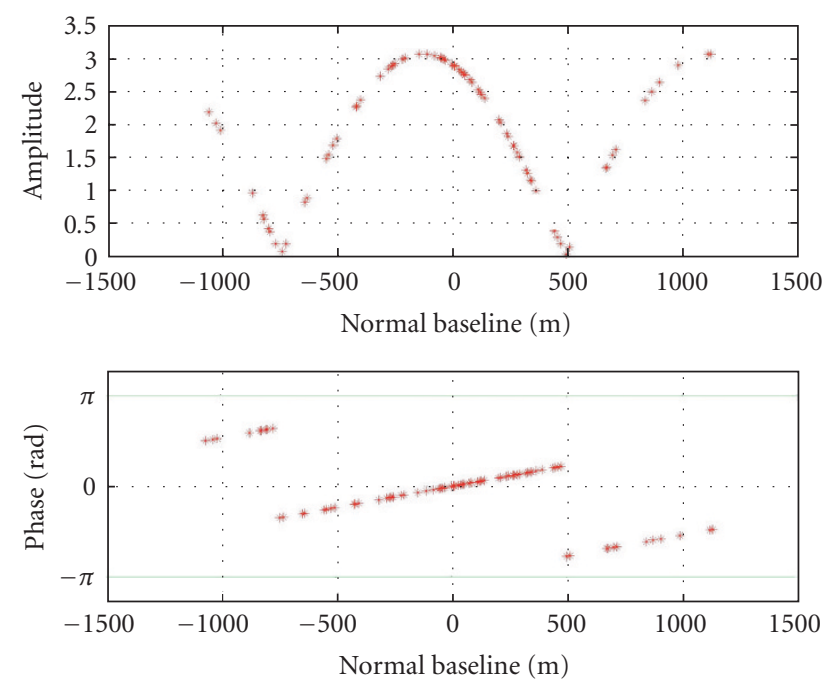

FIgURE 4: Simulated interferometric data (second order). Parameters: $v=0, x_{1}=15 \mathrm{~m}, x_{2}=-4 \mathrm{~m}$, and $\left|z_{1}\right|=\left|z_{2}\right|$. While amplitude data show significant variations, phase values can be fitted using a first-order model.

or in general a homogeneous flat (with respect to the illuminating wavelength) surface can be well described by the model used in conventional PS analysis and SAR interferometry (at least limiting the analysis to phase values). On the contrary, whenever the energy backscattered by the radar targets within the resolution cell is not exactly the same (and none of them dominates the scenario), a distortion of the linear phase trend is introduced and the first-order model may fail to model the data correctly (Figure 5). The phase distortion is a function of the normal baseline distribution of the dataset, the distance between the two scattering centres, and the ratio of the two reflectivity amplitudes.

In general, working on real data, higher-order scattering models should better describe the reflectivity of the image pixels, however their effectiveness depends very much on the distribution of the baseline values, the extension of the resolution cell, and the characteristics of the area of interest.

\subsection{Monte Carlo determination of the rejection region}

Apart from testing the implementation of the two algorithms for data analysis, numerical simulations were used to determine the rejection region for model order selection (Section 3.1).

As already mentioned, a reasonable criteria is to accept $H_{1}$ (i.e., the double-scatterer model) only if the dispersion of the phase residues is significantly reduced passing from the first- to the second-order model, that is, running the NLSM on the data. For Gaussian distribution of the phase residues, it can be demonstrated that [14]

$$
\gamma_{q}=e^{-\sigma_{q}^{2} / 2}, \quad q=I, I I,
$$

where $\sigma_{I}$ and $\sigma_{I I}$ are the dispersion of the phase residues with respect to the model adopted. Therefore, the rejection region can be determined using either $\left(\sigma_{I}, \sigma_{I I}\right)$ or $\left(\gamma_{I}, \gamma_{I I}\right)$.
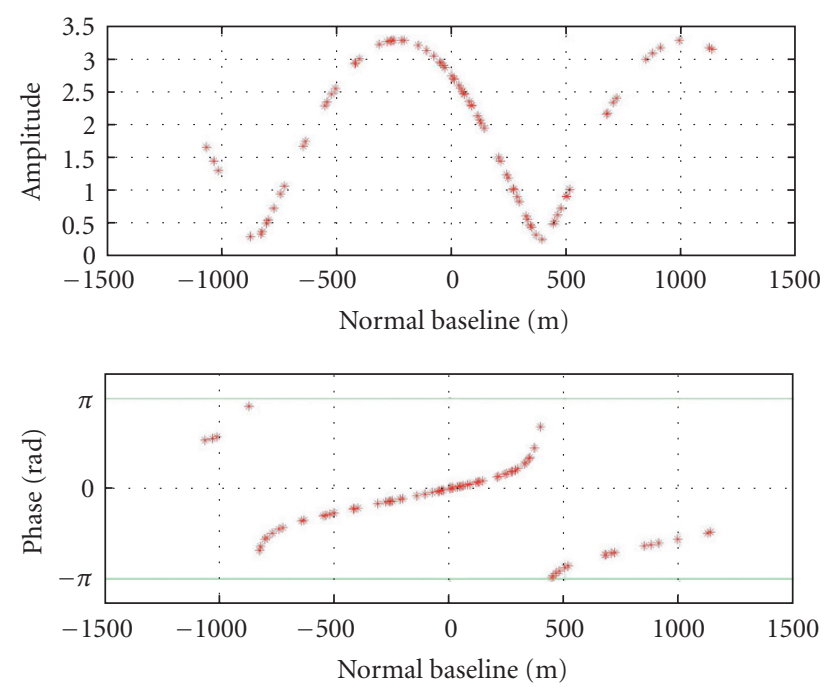

Figure 5: Simulated interferometric data (second order). Parameters: $v=0, x_{1}=15 \mathrm{~m}, x_{2}=-4 \mathrm{~m}$, and $\left|z_{1}\right| \neq\left|z_{2}\right|$. The distortion of the phase values with respect to a data vector generated by a single dominant scatterer (or a homogeneous flat surface) is evident.

A possible operational procedure is then the following. For each of $Q$ different SNR levels, $10^{M}$ realizations of the data vector (sampled at the baseline values of the dataset under study) are created considering a single scattering centre $\left(H_{0}=\right.$ true $)$. The values of $\left(\sigma_{I}, \sigma_{I I}\right)$ are then estimated for each realization and a scatterogram is generated. The rejection region at confidence level $10^{-M}$ can then be determined on the $\left(\sigma_{I}, \sigma_{I I}\right)$-plane by delimiting the cluster of points of the simulation by means of a suitable curve that fits best the edge of the cluster. In Figure 6 the scatterogram obtained after 120000 realizations $(Q=12, M=4)$ of the data vector is reported. Since-in general-we are interested in the detection of radar targets characterized by low phase dispersion (the PS), we can also impose

$$
\sigma_{I I}<\mathrm{THR}
$$

It should be pointed out that the rejection region depends on (1) the number of data available, (2) the distribution of normal baseline values, (3) the parameter space spanned by the estimation algorithms; thus this procedure should be run every time a new dataset has to be processed.

\section{REAL DATA ANALYSIS}

The algorithms described in the previous sections have been applied to a set of 82 SAR scenes acquired by the ERS- 1 and ERS-2 satellites (operated by the European Space Agency) between 1992 and 2003 over Milano, Italy (Frame 2691-Track 208). See in Figure 8 the incoherent average of the SAR data of the area. The distribution of the geometrical and temporal baseline of the acquisitions is reported in Figure 7 where it is possible to appreciate that most of the interferograms have actually baseline values lower than $\sim 500 \mathrm{~m}$. The processed 


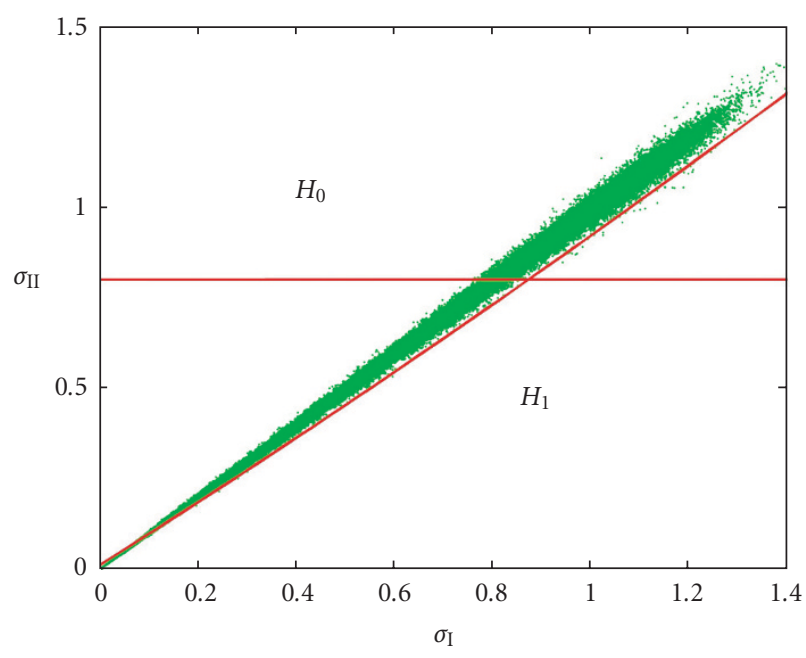

FIGURE 6: Numerical determination of the rejection region for hypothesis testing. 120000 realizations $(Q=12, M=4)$ of the datavector have been generated and both first- and second-order estimation algorithms have been applied. $\sigma_{I}$ and $\sigma_{I I}$ indicate the standard deviation of the phase residues after the application of the firstand second-order estimation algorithms, respectively. The white area specifies the rejection region where $H_{0}$ hypothesis should be rejected.

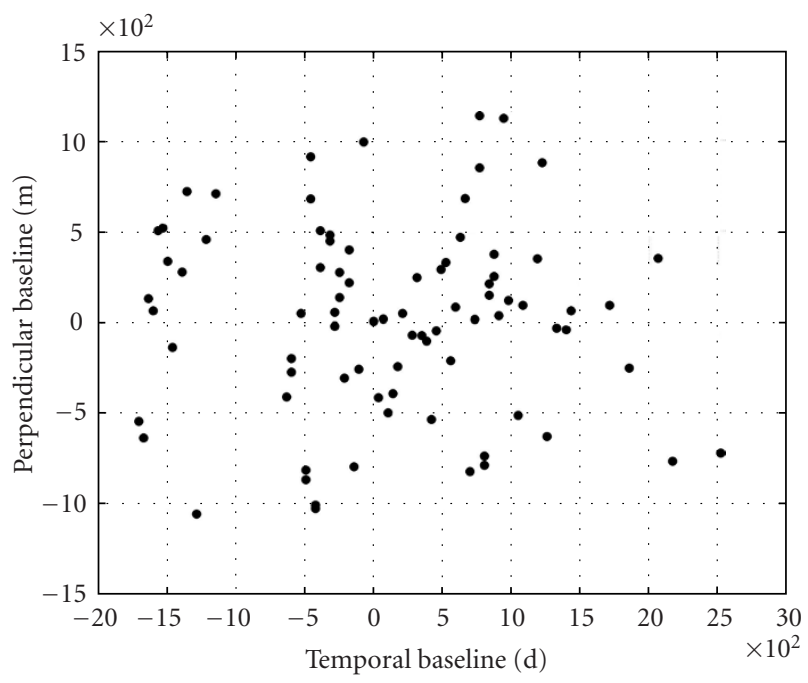

FIGURE 7: Distribution of the geometrical and temporal baseline of the interferometric dataset (SAR data: ESA-ERS. Frame 2691-Track 208).

area is a cut out of the whole scene about $40 \mathrm{~km}^{2}$ wide and it is not affected by significant surface deformation phenomena, apart from terrain subsidence at low rates $(<3 \mathrm{~mm} / \mathrm{yr})$ in some suburbs of the city. The constant velocity model is then suitable for the PS analysis. The density of buildings and man-made structures is extremely high, allowing one to get meaningful statistical parameters for PS characterization in urban areas.

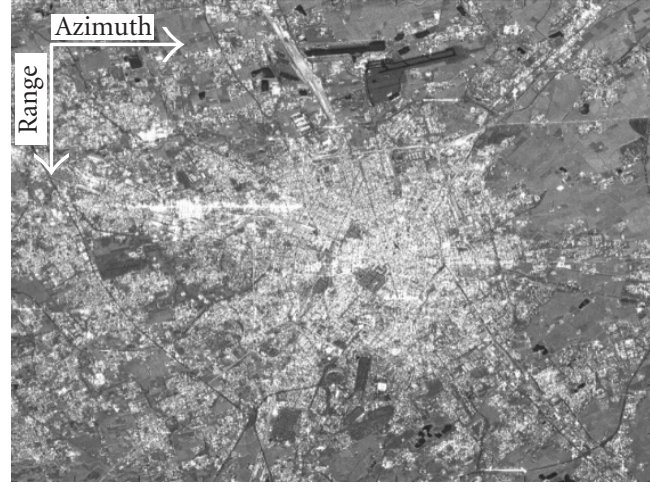

FIGURE 8: Incoherent average of the SAR data available over Milano. Eighty-two images acquired by the ESA-ERS sensors have been processed (Frame 2691-Track 208). The area is heavily urbanized.

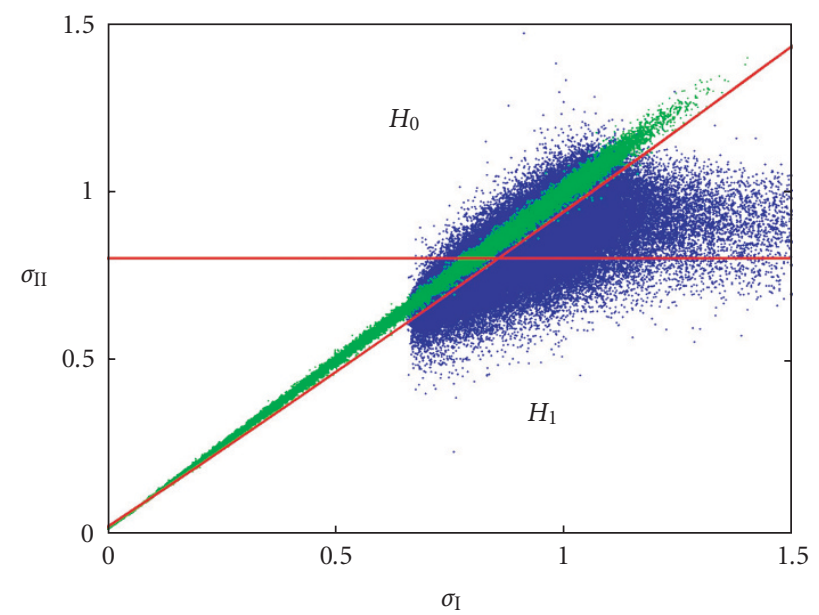

Figure 9: Scatterogram obtained by processing the Milano dataset superimposed on that obtained from the numerical simulation (Figure 5).

Following the algorithm outlined in the previous sections, we applied both first- and second-order analysis tools for data analysis. In order to limit the computational load, second-order model was used only if $\gamma_{I}(P)<0.8$ (since otherwise the pixel was already labelled as PS) and first-order results (i.e., deformation rate $\hat{v}_{I}$, elevation $\hat{\varepsilon}_{I}$, and the firstorder coherence $\hat{\gamma}_{I}$ ) were used to define the portion of the parameter space to be spanned by the NLSM. More precisely, whenever $\gamma_{I}(P)>0.6$,

$$
\begin{aligned}
& v_{I I}=v_{I}+\alpha_{1}, \quad \alpha_{1} \in[-0.75,0.75], \Delta_{v}=0.25 \mathrm{~mm} / \mathrm{yr}, \\
& \varepsilon_{I I, 1}=\varepsilon_{I}+\alpha_{2}, \quad \alpha_{2} \in[-20,20], \Delta_{\varepsilon_{1}}=0.5 \mathrm{~m}, \\
& \varepsilon_{I I, 2}=\varepsilon_{I}+\alpha_{3}, \quad \alpha_{3} \in[-20,20], \Delta_{\varepsilon_{2}}=0.5 \mathrm{~m},
\end{aligned}
$$

where $\Delta$ is the sampling step used in the scanning.

The results obtained at the end of the analysis are reported in Figure 9, where $\left(\sigma_{I}, \sigma_{I I}\right)$ pairs have been superimposed on the rejection region obtained via numerical 


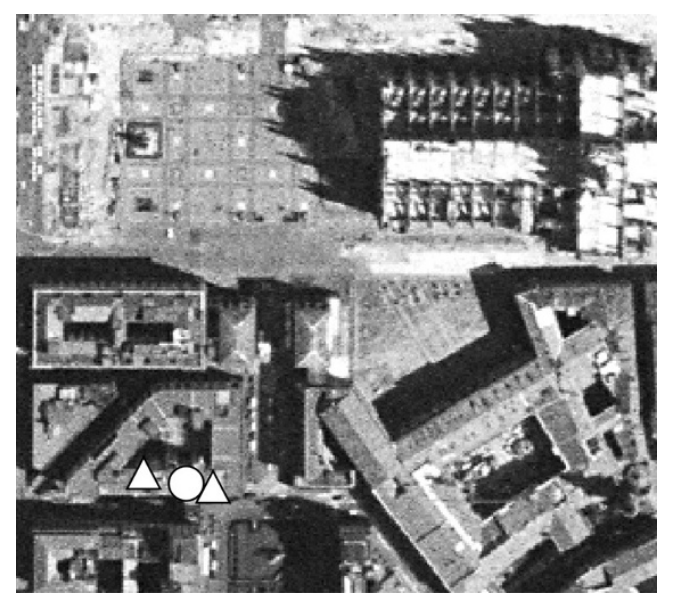

FIGURE 10: Double-scatterer position (triangular symbols) and single PS (circular symbol) superimposed on an orthophoto of Milano.

simulation. 38549 double scatterers were identified (falling in $H_{1}$ ) out of 254226 pixels analysed (15\%). It should be pointed out that since the standard analysis allowed the detection of about 200000 pixels exhibiting $\hat{\gamma}_{I}>0.8$ and the increase in PS density by applying the second-order model was about $20 \%$.

After data processing, double-scatterers positions were estimated (using standard geocoding algorithms) and superimposed on an orthophoto of the area of interest. Two examples are reported in Figures 10 and 11, where the position of the dominant scatterer estimated by the first order analysis (circular symbol) has been superimposed to the positions of the two scattering-centres estimated by the second-order model (triangular symbol). In general, all the positions of the scattering-centres turned out to fall within the shape of building and other structures, but of course more in depth analyses should assess the precision of the estimated parameters.

The improvement in data modelling due to the introduction of the second-order model can be appreciated in Figures 12 and 13 , where the comparison of the two displacement time series relative to scatterers depicted in Figures 10 and 11 is reported.

\section{CONCLUSIONS}

In this paper we have described a possible strategy to improve the performance of the PS technique, using the same multiinterferogram framework and adopting more complex models for the scattering mechanism within the SAR resolution cell. The preliminary results reported here should be considered only as a first contribution toward a full exploitation of multibaseline satellite datasets characterized by high normal baseline values for surface deformation monitoring.

An important issue to be further studied is the statistical distribution of the cross-range dimension of the PS, both in urban and nonurban areas. In fact, the increase in PS density

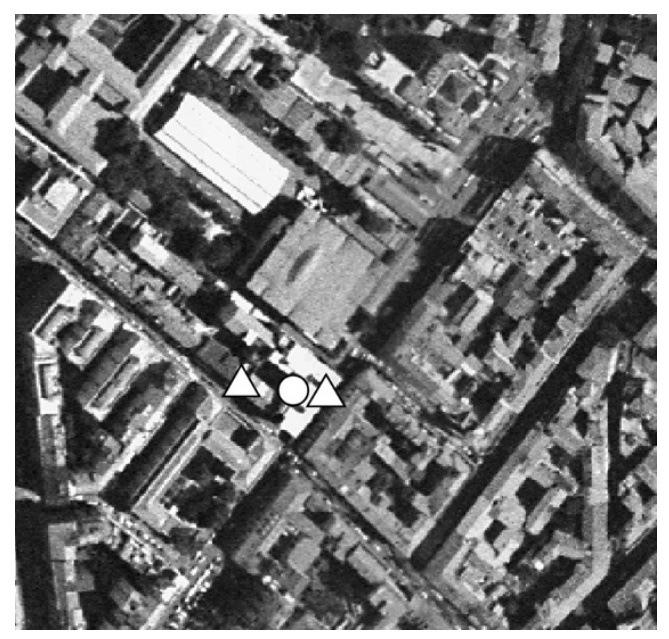

FIGURE 11: Double-scatterer position (triangular symbols) and single PS (circular symbol) superimposed on an orthophoto of Milano.

(20\%), although not negligible, could not completely change the scenario obtained by means of the standard PS analysis, at least in the dataset used in this paper (to a certain extent, this justifies the success of the analysis presented in $[12,13])$. Indeed, since the statistical distribution of the normal baseline values of a typical satellite dataset is far from uniform [14] (within the satellite dead-band), the application of the simple first-order model can be extremely effective. Preliminary results seem to suggest that this kind of algorithms, due to the increased computational load, should be applied only whenever it is mandatory to extract as much information as possible, pushing the technology to its theoretical limits, or-in general-when the first-order PS distribution is not enough for the application at hand.

Future research activities will be also devoted to the application of higher-order models to nonurban areas, assessing the possibility to resolve layover areas (at least where temporal decorrelation is low enough) and the implementation of a more general $K$-order analysis tool, carefully selecting the best algorithm for the estimation of the unknown parameters.

Finally, further efforts should be devoted to precision assessment, trying to cross-validate, using independent data and possible in situ surveys, the parameters estimated from the multibaseline datasets. Even considering higher-order models, the mathematical framework related to a single master image, that is, the generation of all interferogram with respect to the same master acquisition, seems to be extremely effective also to get quantitative estimation of precision and accuracy of the unknown geophysical parameters that can be recovered.

\section{APPENDIX \\ FORMULATION OF THE SECOND-ORDER MODEL}

As mentioned in Section 2.2, the case of two dominant scatterers within the same resolution cell has a mathematical 


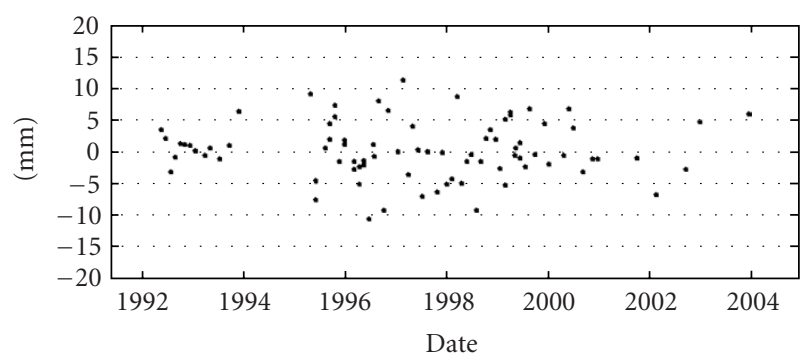

(a)

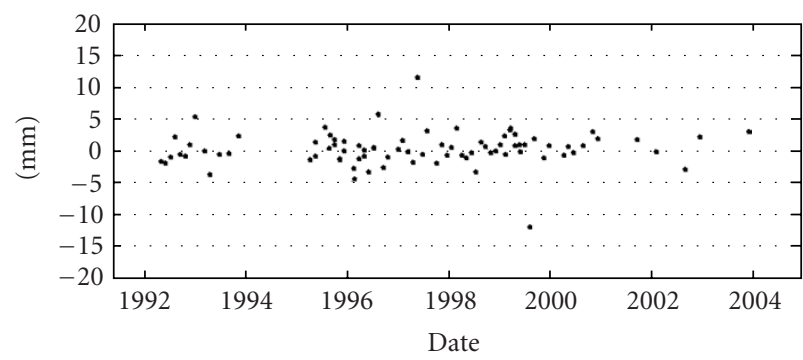

(b)

FIGURE 12: Comparison of the displacement time series estimated by applying (a) first- and (b) second-order models for the PS (double-scatterer) highlighted in Figure 9. $\gamma_{I}=0.62, \gamma_{I I}=0.87$.

framework similar to the one used in the direction-of-arrival analysis $[23,24,26]$.

Adopting the first Born approximation (and considering valid the superposition of the effects), the signal received by the $i$ th sensor $(i=1, \ldots, N)$ can be written as

$$
s_{i}=z_{1}^{j(4 \pi / \lambda) r_{1 i}} e^{j C_{v}^{i} v}+z_{2}^{j(4 \pi / \lambda) r_{2 i}} e^{j C_{v}^{i} v}
$$

$r_{k i}$ being the target-sensor distance, $z_{k}$ the complex reflectivity of the $k$ th scattering centre $(k=1,2)$, and $C_{v}^{i}=4 \pi t_{i} / \lambda$. where $t_{i}$ is the $i$ th the temporal baseline.

From Figure 1, the approximated target-sensor distance $r_{k i}$ reads

$$
\begin{aligned}
r_{k i}=r\left(B_{i}, t_{i}, x_{k}\right) & =\sqrt{R_{M}^{2}+\left(B_{i}-x_{k}\right)^{2}} \\
& \cong R_{M}+\frac{\left(B_{i}-x_{k}\right)^{2}}{2 R_{M}}, \quad k=1,2 .
\end{aligned}
$$

Moreover, hypothesis (7) remarks that all the scatterers within the same resolution cell are supposed to be affected by the same displacement:

$$
v_{1}=v_{2}=v
$$

The backscattered signal acquired by the master acquisition and the $i$ th slave acquisition yields to the computation of the

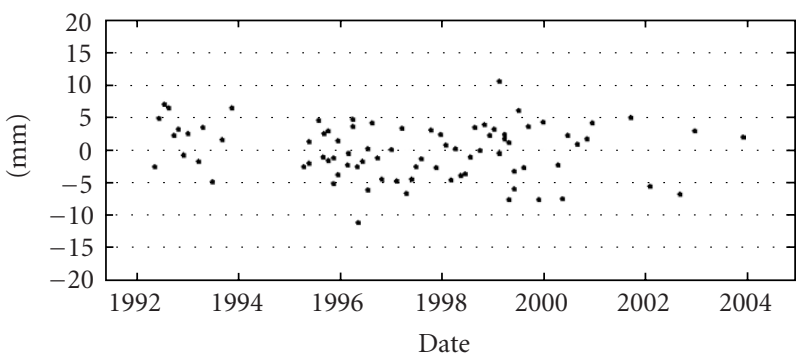

(a)

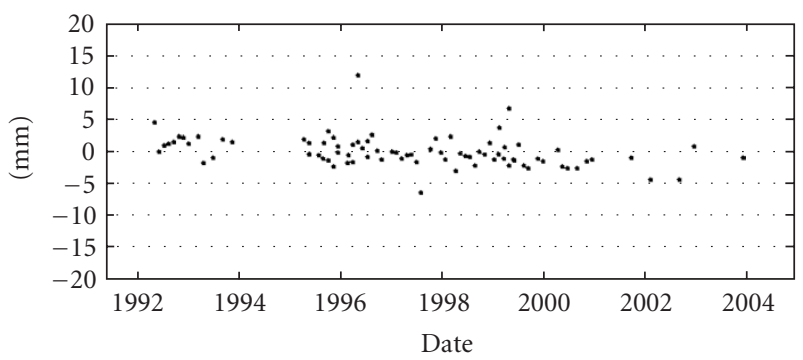

(b)

FIGURE 13: Comparison of the displacement time series estimated by applying (a) first- and (b) second-order models for the PS (double-scatterer) highlighted in Figure 12. $\gamma_{I}=0.66, \gamma_{I I}=0.90$.

$i$ th interferogram $Y_{i}$ (divided by the amplitude of the master scene) as

$$
\begin{aligned}
Y_{i}=\frac{s_{M} s_{i}^{*}}{\left|s_{M}\right|} & \\
=\frac{e^{-j C_{v}^{i v} v}}{\left|s_{M}\right|} & \left.|| z_{1}\right|^{2} e^{j(4 \pi / \lambda)\left(\left(B_{n_{i}} x_{1} / R_{M}\right)-\left(B_{n_{i}}^{2} / 2 R_{M}\right)\right)} \\
& +z_{1} z_{2}^{*} e^{j(4 \pi / \lambda)\left(\left(x_{1}^{2} / 2 R_{M}\right)-\left(x_{2}^{2} / 2 R_{M}\right)+\left(B_{n_{i}} x_{2} / R_{M}\right)-\left(B_{n_{i}}^{2} / 2 R_{M}\right)\right)} \\
& +z_{1}^{*} z_{2} e^{j(4 \pi / \lambda)\left(\left(x_{2}^{2} / 2 R_{M}\right)-\left(x_{1}^{2} / 2 R_{M}\right)+\left(B_{n_{i}} x_{1} / R_{M}\right)-\left(B_{n_{i}}^{2} / 2 R_{M}\right)\right)} \\
& \left.+\left|z_{2}\right|^{2} e^{j(4 \pi / \lambda)\left(\left(B_{n_{i}} x_{2} / R_{M}\right)-\left(B_{n_{i}}^{2} / 2 R_{M}\right)\right)}\right] .
\end{aligned}
$$

The $i$ th differential interferogram (computed using the a priori elevation $x=0$-Figure 2) is then obtained by the following expression:

$$
y_{i} \equiv \frac{s_{M} s_{i}^{*}}{\left|s_{M}\right|} e^{-j(4 \pi / \lambda)\left(R_{M}-R_{i}\right)}=Y_{i} e^{-j(4 \pi / \lambda)\left(R_{M}-R_{i}\right)},
$$

with

$$
R_{i}=\sqrt{R_{M}^{2}+B_{n_{i}}^{2}} \cong R_{M}+\frac{B_{n_{i}}^{2}}{2 R_{M}},
$$


where the approximation is valid for satellite sensors $\left(R_{M}>\right.$ $800 \mathrm{~km}$ and $|B|<2 \mathrm{~km})$.

By substitution of (A.4) and (A.6) in (A.5) and using relation (2),

$$
\begin{aligned}
y_{i}= & e^{-j C_{v}^{i} v} e^{j A_{\mathrm{DEM}}^{i} x_{1}}\left[\frac{\left|z_{1}\right|^{2}+z_{2} z_{1}^{*} e^{j(4 \pi / \lambda)\left(x_{2}^{2}-x_{1}^{2} / 2 R_{M}\right)}}{\left|s_{M}\right|}\right] \\
& +e^{-j C_{v}^{i} v} e^{j A_{\mathrm{DEM}}^{i} x_{2}}\left[\frac{\left|z_{2}\right|^{2}+z_{1} z_{2}^{*} e^{j(4 \pi / \lambda)\left(x_{1}^{2}-x_{2}^{2} / 2 R_{M}\right)}}{\left|s_{M}\right|}\right] .
\end{aligned}
$$

Now we define the complex expressions $\beta_{1}$ and $\beta_{2}$ as follows:

$$
\begin{aligned}
& \beta_{1}=\frac{\left|z_{1}\right|^{2}+z_{2} z_{1}^{*} e^{j(4 \pi / \lambda)\left(x_{2}^{2}-x_{1}^{2} / R_{M}\right)}}{\left|s_{M}\right|}, \\
& \beta_{2}=\frac{\left|z_{2}\right|^{2}+z_{1} z_{2}^{*} e^{j(4 \pi / \lambda)\left(x_{1}^{2}-x_{2}^{2} / R_{M}\right)}}{\left|s_{M}\right|} .
\end{aligned}
$$

Using now (A.8) in (A.7) we finally obtain the definition of the second-order model of the differentials:

$$
\begin{aligned}
y_{i} & =\beta_{1} e^{j\left(A_{\mathrm{DEM}}^{i} x_{1}-C_{v}^{i} v\right)}+\beta_{2} e^{j\left(A_{\mathrm{DEM}}^{i} x_{2}-C_{v}^{i} v\right)} \\
& =\beta_{1} e^{j\left(C_{\mathrm{DEM}}^{i} \varepsilon_{1}-C_{v}^{i} v\right)}+\beta_{2} e^{j\left(C_{\mathrm{DEM}}^{i} \varepsilon_{2}-C_{v}^{i} v\right)}, \quad i=1, \ldots, N,
\end{aligned}
$$

where $x=\varepsilon / \sin \theta$ and $A_{\mathrm{DEM}}^{i}=C_{\mathrm{DEM}}^{i} \sin \theta$.

Starting from (A.8) and computing expressions (A.1) and (A.2) for the master acquisition $\left(C_{v}^{M}=0\right.$, with $t_{M}=0$, and $B_{M}=0$ ), it can be demonstrated that

$$
\left|\beta_{1}\right|^{2}=\frac{\left|z_{1}\right|^{2}\left(\left|z_{1}\right|^{2}+\left|z_{2}\right|^{2}+2\left|z_{1}\right|\left|z_{2}\right| \cos \xi\right)}{\left|z_{1}\right|^{2}+\left|z_{2}\right|^{2}+2\left|z_{1}\right|\left|z_{2}\right| \cos \xi}=\left|z_{1}\right|^{2}
$$

where $\xi=(4 \pi / \lambda)\left(x_{2}^{2}-x_{1}^{2} / 2 R_{M}\right)+\Delta \psi$, with $\Delta \psi$ the difference between the two scatterers reflectivity phases. Similarly, for $\beta_{2}$,

$$
\left|\beta_{2}\right|^{2}=\frac{\left|z_{2}\right|^{2}\left(\left|z_{2}\right|^{2}+\left|z_{1}\right|^{2}+2\left|z_{1}\right|\left|z_{2}\right| \cos \xi\right)}{\left|z_{2}\right|^{2}+\left|z_{1}\right|^{2}+2\left|z_{1}\right|\left|z_{2}\right| \cos \xi}=\left|z_{2}\right|^{2}
$$

Equations (A.8) and (A.9) correspond to the second-order model described in Section 2.2 through (12) and (14).

\section{ACKNOWLEDGMENTS}

Authors wish to thank the whole technical staff of TRE for the implementation of the algorithms described in this paper. This research was self-financed by TRE and Politecnico di Milano.

\section{REFERENCES}

[1] A. K. Gabriel, R. M. Goldstein, and H. A. Zebker, "Mapping small elevation changes over large areas: differential radar interferometry," Journal of Geophysical Research, vol. 94, no. B7, pp. 9183-9191, 1989.

[2] D. Massonnet and K. L. Feigl, "Radar interferometry and its application to changes in the earth's surface," Reviews of Geophysics, vol. 36, no. 4, pp. 441-500, 1998.

[3] P. A. Rosen, S. Hensley, I. R. Joughin, et al., "Synthetic aperture radar interferometry," Proc. IEEE, vol. 88, no. 3, pp. 333$382,2000$.

[4] R. Bürgmann, P. A. Rosen, and E. J. Fielding, "Synthetic aperture radar interferometry to measure Earth's surface topography and its deformation," Annual Review of Earth and Planetary Sciences, vol. 28, pp. 169-209, May 2000.

[5] R. F. Hanssen, Radar Interferometry: Data Interpretation and Error Analysis, Kluwer Academic, Dordrecht, the Netherlands, 2001.

[6] S. Madsen, H. A. Zebker, and J. Martin, "Topographic mapping using radar interferometry: processing techniques," IEEE Trans. Geosci. Remote Sensing, vol. 31, no. 1, pp. 246-256, 1993.

[7] D. Massonnet, P. Briole, and A. Arnaud, "Deflation of Mount Etna monitored by spaceborne radar interferometry," Nature, vol. 375, no. 6532, pp. 567-570, 1995.

[8] D. Massonnet, K. L. Feigl, M. Rossi, and F. Adragna, "Radar interferometric mapping of deformation in the year after the Landers earthquake," Nature, vol. 369, no. 6477, pp. 227-230, 1994.

[9] D. Massonnet, W. Thatcher, and H. Vadon, "Detection of postseismic fault-zone collapse following the Landers earthquake," Nature, vol. 382, no. 6592, pp. 612-616, 1996.

[10] G. Peltzer and P. A. Rosen, "Surface displacement of the 17 May 1993 Eureka Valley, California earthquake observed by SAR interferometry," Science, vol. 268, pp. 1333-1336, 1995.

[11] H. A. Zebker and J. Villasenor, "Decorrelation in interferometric radar echoes," IEEE Trans. Geosci. Remote Sensing, vol. 30, no. 5, pp. 950-959, 1992.

[12] A. Ferretti, C. Prati, and F. Rocca, "Permanent scatterers in SAR interferometry," IEEE Trans. Geosci. Remote Sensing, vol. 39, no. 1, pp. 8-20, 2001.

[13] A. Ferretti, C. Prati, and F. Rocca, "Nonlinear subsidence rate estimation using permanent scatterers in differential SAR interferometry," IEEE Trans. Geosci. Remote Sensing, vol. 38, no. 5, pp. 2202-2212, 2000.

[14] C. Colesanti, A. Ferretti, F. Novali, C. Prati, and F. Rocca, "SAR monitoring of progressive and seasonal ground deformation using the permanent scatterers technique," IEEE Trans. Geosci. Remote Sensing, vol. 41, no. 7, pp. 1685-1701, 2003.

[15] P. Berardino, G. Fornaro, R. Lanari, and E. Sansosti, "A new algorithm for surface deformation monitoring based on small baseline differential SAR interferograms," IEEE Trans. Geosci. Remote Sensing, vol. 40, no. 11, pp. 2375-2383, 2002.

[16] O. Mora, J. J. Mallorqui, and A. Broquetas, "Linear and nonlinear terrain deformation maps from a reduced set of interferometric SAR images," IEEE Trans. Geosci. Remote Sensing, vol. 41, no. 10, pp. 2243-2253, 2003.

[17] S. Usai, "A least squares database approach for SAR interferometric data," IEEE Trans. Geosci. Remote Sensing, vol. 41, no. 4, pp. 753-760, 2003.

[18] D. T. Sandwell and E. J. Price, "Phase gradient approach to stacking interferograms," Journal of Geophysical Research, vol. 103, no. B12, pp. 30183-30204, 1998. 
[19] S. Salvi, S. Atzori, C. Tolomei, et al., "Inflation rate of the Colli Albani volcanic complex retrieved by the permanent scatterers SAR interferometry technique," Geophysical Research Letters, vol. 31, no. 21, 2004.

[20] G. E. Hilley, R. Bürgmann, A. Ferretti, F. Novali, and F. Rocca, "Dynamic of slow-moving landslides from permanent scatterer analysis," Science, vol. 304, no. 5679, pp. 1952-1955, 2004.

[21] A. Reigber and A. Moreira, "First demonstration of airborne SAR tomography using multibaseline L-band data," IEEE Trans. Geosci. Remote Sensing, vol. 38, no. 5, pp. 2142-2152, 2000.

[22] C. R. Smith and P. M. Goggans, "RADAR target identification," IEEE Antennas Propagat. Mag., vol. 35, no. 2, pp. 27-38, 1993.

[23] F. Gini, F. Lombardini, and M. Montanari, "Layover solution in multibaseline SAR interferometry," IEEE Trans. Aerosp. Electron. Syst., vol. 38, no. 4, pp. 1344-1356, 2002.

[24] F. Lombardini, M. Montanari, and F. Gini, "Reflectivity estimation for multibaseline interferometric radar imaging of layover extended sources," IEEE Trans. Signal Processing, vol. 51, no. 6, pp. 1508-1519, 2003.

[25] M. Lorenzi and D. Magni, "Sviluppi della tecnica dei Bersagli Permanenti," M. Sc. Thesis, Politecnico di Milano University, Milano, Italy, 2001.

[26] H. Krim and M. Viberg, "Two decades of array signal processing research: the parametric approach," IEEE Signal Processing Mag., vol. 13, no. 4, pp. 67-94, 1996.

[27] P. Stoica and R. L. Moses, Introduction to Spectral Analysis, Prentice-Hall, Englewood Cliffs, NJ, USA, 1997.

[28] S. M. Kay, Fundamentals of Statistical Signal Processing, Volume I: Estimation Theory, Prentice-Hall, Englewood Cliffs, NJ, USA, 1993.

[29] H. Akaike, "Information theory and an extension of the maximum likelihood principle," in Proc. IEEE 2nd International Symposium on Information Theory, B. N. Petrov and F. Caski, Eds., pp. 267-281, Akademiai Kiado, Ashkelon, Israel, June 1973.

[30] J. Rissanen, "Modelling by the shortest data description," Automatica, vol. 14, no. 5, pp. 465-471, 1978.

Alessandro Ferretti was born in Milano, Italy, on January 27, 1968. He received the degree in electrical engineering (cum laude) from Politecnico di Milano (POLIMI) in 1993 and the M.S. degree in information technology (cum laude) from CEFRIEL. In May 1994 he joined the POLIMI Radar Group working on SAR interferometry. In July 1997, he received the Ph.D. degree in electrical engineering from POLIMI. After devoting most of his research efforts to multitemporal SAR data stacks at the Department of Electronics, he developed together with Professors Rocca and Prati what is now called the "permanent scatterer technique," a technology patented in 1999 that can overcome most of the difficulties encountered in conventional SAR interferometry. In March 2000 he founded, together with Professors Rocca and Prati, and Politecnico di Milano the company TeleRilevamento Europa (TRE), the first POLIMI spin-off, where he is currently the Managing Director. Dr. Ferretti has been involved in many projects financed by the European Space Agency and was the promoter, in 2003, of the first interferometric archive of Radarsat data on a national level. His research interests include radar data processing, optimization algorithms, data fusion, and the use of remote sensing information for civil protection applications.
Marco Bianchi was born in 1978 in Busto Arsizio, Italy. He received a Laurea degree in telecommunication engineering from Politecnico di Milano (POLIMI) in 2003 with a study about phase ambiguity estimation in SAR interferometry developed at Delft University of Technology, The Netherlands. In the same year, he joined Tele-Rilevamento Europa, Milano, Italy, working on the permanent scatterers technique, mainly focusing his research activity on the higher-order models for PS analysis.

Claudio Prati was born in Milano on March 20, 1958. He is Full Professor of telecommunications at the Electronic Department, Politecnico di Milano. He holds three patents on the SAR images processing. He has been awarded two prizes from the IEEE Geoscience and Remote Sensing Society (IGARSS'89 and IGARSS'99). He published more than 100 papers on SAR data processing and interferometry. $\mathrm{He}$ is cofounder of Tele-Rilevamento Europa (TRE), an RS spin-off company of POLIMI.

Fabio Rocca received his degree in electrical engineering in 1962. He is a Professor of digital signal processing at Politecnico di Milano. He dedicated his research to digital signal processing for television bandwidth compression, emission tomography, seismic data processing, and SAR. He was a Visiting Professor at Stanford University several times from 1978 to $1987 / 88$, Department Chairman in 1975-1978, Com-

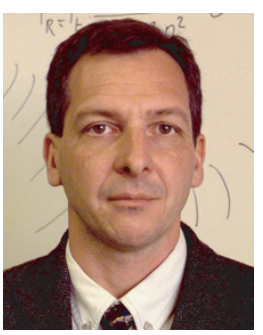
missione d'Ateneo 1980-1993, cofounder of two small technological companies, Tele-Rilevamento Europa and Aresys, spin-off Politecnico di Milano, President of OGS Trieste (1982-1983), Coordinator of the first EEC research program in Geosciences, Member of the Scientific Council of IN-OGS and of the SAG for TERRASAR-L of ESA, past President of the EAEG, and Honorary Member of the SEG (1989) and EAGE (1998). He received the HUSPI Award in 1979, IGARSS Awards for 1989 and 1999, Schlumberger Award for 1990, Italgas Award for Telecommunications in 1995, Special SEG Commendation Award in 1998, Eduard Rhein Foundation Technology Award for 1999, Doctor Honoris Causa in Geophysics from the Institut Polytechnique de Lorraine in 2001, EUSAR Best Paper Award in 2004. He is an Associate Editor of Journal of Seismic Exploration and in the Editorial Committee of the Oil \& Gas Science and Technology (Revue de l' Institut Français du Pétrole). 